\title{
Breath gas metabolites and bacterial metagenomes from cystic fibrosis airways indicate active $\mathrm{pH}$ neutral 2,3-butanedione fermentation
}

\author{
Katrine L Whiteson ${ }^{1}$, Simone Meinardi ${ }^{2}$, Yan Wei Lim ${ }^{1}$, Robert Schmieder ${ }^{1}$, \\ Heather Maughan ${ }^{3}$, Robert Quinn ${ }^{1}$, Donald R Blake ${ }^{2}$, Douglas Conrad ${ }^{4}$ and Forest Rohwer ${ }^{1}$ \\ ${ }^{1}$ Department of Biology, San Diego State University, San Diego, CA, USA; ${ }^{2}$ Department of Chemistry, \\ University of California, Irvine, CA, USA ${ }^{3}$ Ronin Institute, Montclair, NJ, USA and ${ }^{4}$ Department of Medicine, \\ University of California, San Diego, La Jolla, CA, USA
}

\begin{abstract}
The airways of cystic fibrosis (CF) patients are chronically colonized by patient-specific polymicrobial communities. The conditions and nutrients available in CF lungs affect the physiology and composition of the colonizing microbes. Recent work in bioreactors has shown that the fermentation product 2,3-butanediol mediates cross-feeding between some fermenting bacteria and Pseudomonas aeruginosa, and that this mechanism increases bacterial current production. To examine bacterial fermentation in the respiratory tract, breath gas metabolites were measured and several metagenomes were sequenced from CF and non-CF volunteers. 2,3-butanedione was produced in nearly all respiratory tracts. Elevated levels in one patient decreased during antibiotic treatment, and breath concentrations varied between CF patients at the same time point. Some patients had high enough levels of 2,3-butanedione to irreversibly damage lung tissue. Antibiotic therapy likely dictates the activities of 2,3-butanedione-producing microbes, which suggests a need for further study with larger sample size. Sputum microbiomes were dominated by $P$. aeruginosa, Streptococcus spp. and Rothia mucilaginosa, and revealed the potential for 2,3-butanedione biosynthesis. Genes encoding 2,3-butanedione biosynthesis were disproportionately abundant in Streptococcus spp, whereas genes for consumption of butanedione pathway products were encoded by $P$. aeruginosa and R. mucilaginosa. We propose a model where low oxygen conditions in CF lung lead to fermentation and a decrease in $\mathrm{pH}$, triggering 2,3-butanedione fermentation to avoid lethal acidification. We hypothesize that this may also increase phenazine production by $P$. aeruginosa, increasing reactive oxygen species and providing additional electron acceptors to CF microbes.

The ISME Journal (2014) 8, 1247-1258; doi:10.1038/ismej.2013.229; published online 9 January 2014

Subject Category: Integrated genomics and post-genomics approaches in microbial ecology

Keywords: breath gas; cystic fibrosis; metagenomics; polymicrobial infection; metabolomics; biomarker
\end{abstract}

\section{Introduction}

Cystic fibrosis (CF) is a life-threatening inherited disease most prominent among Caucasians (affecting 1 in 3200 births), and is characterized by destruction of pulmonary structure and function arising from chronic airway polymicrobial infections. Life expectancy for CF patients has risen from infancy to nearly 40 years since the 1960s (Strausbaugh and Davis, 2007). Despite this progress, maintaining lung function remains problematic. The inability to clear abnormal, thick mucus from lung airways promotes chronic infection, and requires time-consuming

Correspondence: KL Whiteson, Biology Department LS301, San Diego State University, 5500 Campanile Drive, San Diego, CA 92182, USA.

E-mail: katrinewhiteson@gmail.com

Received 5 September 2013; revised 14 November 2013; accepted 15 November 2013; published online 9 January 2014 treatments to physically loosen and remove mucus from the airways. Starting as early as childhood, CF patients develop long-term bacterial infections characterized by the presence of opportunistic pathogens, such as Pseudomonas aeruginosa. Daily symptoms include increased sputum production and cough, punctuated by intermittent periods of more severe symptoms referred to as pulmonary exacerbations (Bilton et al., 2011). A poorly understood combination of changes in microbial infection and inflammation causes exacerbations, which are difficult to define or diagnose (Goss and Burns, 2007). Lung function is usually restored toward pre-exacerbation levels after several days of intravenous antibiotic administration. Each exacerbation is thought to cause irreversible scarring and damage to lung tissue (Sanders et al., 2010, 2011).

Microbes living in the CF lung persist in the nutrient rich mixture of abnormally thick mucus, extracellular DNA, cell debris and toxic immune 
molecules (Conrad et al., 2012; Lynch and Bruce, 2013). Decreasing oxygen availability toward the center of sputum drives some fraction of the microbial population to fermentation and anaerobic respiration (Worlitzsch et al., 2002; Yoon et al., 2002). The fluid lining the airways in CF has a lower $\mathrm{pH}$ relative to healthy tissue, even before microbial colonization (Pezzulo et al., 2012), likely because of impaired bicarbonate transport, and during exacerbation $\mathrm{pH}$ has been shown to drop even further in exhaled breath condensate samples (Tate et al., 2002; Ojoo et al., 2005). Oral microbes that can aspirate to the lung are known to produce and tolerate acid in response to fermentation of dietary sugars (McLean et al., 2012). One mechanism employed by microbes to avoid lethal acidification from low $\mathrm{pH}$ fermentation products is an alternative fermentation pathway, known as acetoin metabolism, which produces the $\mathrm{pH}$ neutral and buttery flavored compounds 2,3-butanedione (also known as diacetyl) and 2,3-butanediol (Figure 1) (Bartowsky and Henschke, 2004).

2,3-butanedione has the potential to harm lung tissue and benefit microbes. The basis of its toxicity in the lung may be its reactivity with guanidinium groups such as those found in arginine side chains (Mathews et al., 2010). Reactivity with guanidinium groups also enables colorimetric detection of 2,3-butanedione with the Voges-Proskauer reaction developed in the 1880s (Voges and Proskauer, 1898). Although at high concentrations ( $>1 \mathrm{~mm}$ ),

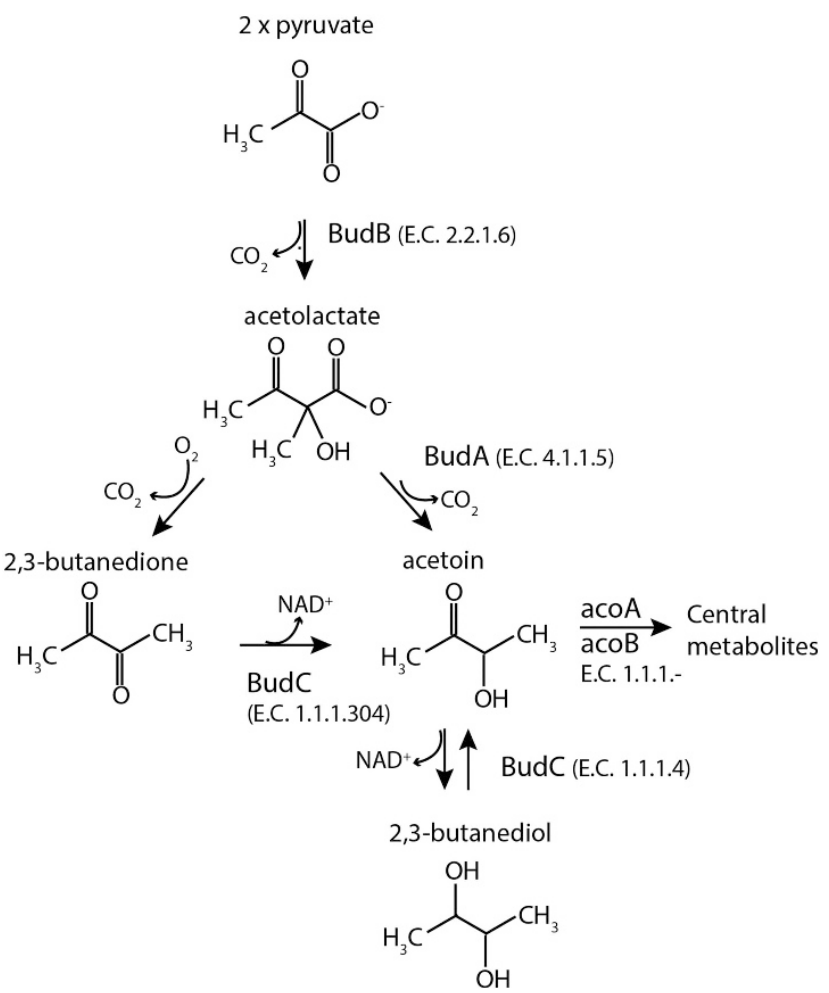

Figure 1 Schematic of pathway for 2,3-butanedione metabolism. 2,3-butanedione has broad antimicrobial activity (Jay, 1982; Kim et al., 2013), lower doses (nм to $\mu \mathrm{M})$ may enhance microbial survival. For example, when E. coli was exposed to 2,3-butanedione emitted by $B$. subtilis, broad changes in gene expression suggesting increased antibiotic resistance and motility were observed (Kim et al., 2013).

If present in the lung, 2,3-butanedione could also impact lung function and the resident microbial community through its effects on the production of toxic redox active molecules known as phenazines. In the presence of oxygen, phenazines generate the deadly oxygen radicals that originally led to their designation as antibiotics. However, at low oxygen tensions found in CF lungs, the phenazines could act as alternative electron acceptors and enable anaerobic respiration for other members of the community, potentially recycling their redox state through contact with occasional oxygen molecules or abundant iron molecules in the CF lung (Wang and Newman, 2008; Wang et al., 2010; Ghio et al., 2012). The last step in the biosynthesis of pyocyanin highlights the relationship between pyocyanin and oxygen, as the conversion of phenazine-1-carboxylic acid to pyocyanin requires oxygen for one of the phenazine-modifying genes (PhzS), a flavin-containing mono-oxygenase. Pyocyanin may serve an important function in low oxygen conditions as an alternative electron acceptor, and yet pyocyanin production depends on the presence of oxygen. This is an interesting puzzle that may relate to the currently under-explored specializations of different phenazines. Each phenazine type has unique properties including redox potential; the ability to transfer electrons to $\mathrm{O}_{2}$ or $\mathrm{Fe}^{3+}$ varies and is dependent on $\mathrm{pH}$ (Wang and Newman, 2008).

Although the main CF pathogen and phenazine producer $P$. aeruginosa is not known to synthesize 2,3-butanedione, it consumes 2,3-butanedione produced by cocultured Staphylococcus aureus (Filipiak et al., 2012b), and responds to 2,3-butanediol produced by cocultured Enterobacter aerogenes by increasing its current production rate and producing more of the phenazine pyocyanin (Venkataraman et al., 2011). Increasing concentrations of pyocyanin along with another phenazine, phenazine-1-carboxylic acid, are associated with disease severity in CF patients (Hunter et al., 2012).

Because these studies have been limited to controlled experimental cultures, we took an ecosystem-level approach that investigated whether the microaerophilic conditions of the CF lung could steer microbial metabolism toward production of 2,3-butanedione. Here we show that 2,3-butanedione is a component of $\mathrm{CF}$ and non-CF breath gas, and identify microbial genes for 2,3-butanedione synthesis in CF and non-CF sputum. 2,3-butanedione, likely to be produced by Streptococcus spp., may lead to the increased phenazine production when consumed by $P$. aeruginosa, a relevant and drastic combination for the health of 
CF patients. We discuss a model where increased production of phenazines offers an alternative electron acceptor, which may enable phenazines to recycle their redox state through donation of electrons to distant oxygen molecules, nitrate or abundant $\mathrm{Fe}^{3+}$ molecules in the lung.

\section{Materials and methods}

Ethical statement

All collection was in accordance with the University of California Institutional Review Board (HRPP 081500) and San Diego State University Institutional Review Board (SDSU IRB\#2121).

\section{Metagenome collection and analysis}

For the longitudinal analysis, breath gases were sampled from one CF patient and one healthy individual at approximately monthly intervals. For the combined cross-sectional analysis of breath gases and metagenomes, induced sputum samples were collected from seven CF patients and one healthy control. Separation of microbial and human cells was achieved through hypotonic lysis of eukaryotic cells, followed by washing and DNA extraction from microbial cells (Lim et al., 2012). Ion Torrent sequencing of these samples yielded 19.8 million sequences. After removing low quality reads with PRINSEQ (Schmieder and Edwards, 2011a) and human sequences using DeconSeq (Schmieder and Edwards, 2011b) with the commands specified in the Supplementary Materials, 13.6 million reads of $\sim 140 \mathrm{bp}$ were retained for further analysis (Supplementary Table 7). Genes related to butanedione synthesis from the SEED database (Aziz et al., 2012) and phenazine synthesis genes from the Patric database (Snyder et al., 2007) were used in BLASTn searches for related sequences in metagenomic data from CF patients. Metagenomic sequences were selected if they matched butanedione and phenazine synthesis pathway genes with a minimum length of $40 \mathrm{bp}$, sequence identity of $40 \%$ and a BLAST $e$-value cutoff of $1 \times 10^{-10}$. Microbial DNA isolated from SF sputum samples as part of a related study (Lim et al., 2012) was also mined for butanedione metabolism related genes. Metatranscriptome data from 454 sequencing of 10 sputum samples from four CF patients (Lim et al., 2012) consisted of 1549273 reads with an average length of $280 \mathrm{bp}$, while 806009 of the reads come from five time-points taken from patient CF1 (Lim et al., 2012). Bacterial mRNAs were not especially abundant in these data sets, which have been published previously (Lim et al., 2012). However, we were able to examine the transcription of the human ferritin gene, as mentioned in the Discussion and Supplementary Information. BLAST hits that were at least $60 \mathrm{bp}$ long with an $e$-value cutoff $1 \times 10^{-5}$ were retained. Several queries were searched against the transcriptome data, including: the heavy and light chain of ferritin (GI:56682958 and GI:56682960) and the two standards (GI:223459775 and GI:183603937) chosen for their transcription rate stability across tissue types (Neville et al., 2011). Raw counts are reported to avoid the potential for false positives demonstrated with the reads per kilobase per million mapped reads measure (Dillies et al., 2012).

Species indicator values were calculated from the metagenome data using the $\mathrm{R}$ package LABDSV (http://cran.r-project.org/web/packages/labdsv/index. html) (R: www.R-project.org). An operational taxonomical unit table with the relative abundance of each genus in each sputum sample was used as input for the 'indval' function. The 'Acute' and 'Suppressive' antibiotic groupings were used to cluster genera for the 'indval' function.

\section{Breath gas sample collection}

At each time point, three breath samples were collected within $5 \mathrm{~min}$ from a single CF patient in a $1.9 \mathrm{~L}$ custom-built electro-polished stainless-steel canister (Kamboures et al., 2005). At the beginning of the second sample, a simultaneous breath sample from a healthy control occupying the same room was taken. At the same time, a background sample of room air was taken by placing a canister within a few feet of the human breath sample donors. Volunteers were instructed to allow the first third of their breath to escape to the room before turning the valve to collect the sample in the canister to avoid collecting breath gases from the oral cavity. Food and drink in the hours before sampling along with health status were recorded; volunteers were instructed to eat and drink normally until $1 \mathrm{~h}$ before sample collection. The analytical system used to analyze the breath samples is similar to the system described in (Colman et al., 2001). Briefly, $235 \pm 3 \mathrm{~cm}^{3}$ (at Standard Temperature and Pressure) of an air sample is pre-concentrated in a stainlesssteel loop filled with glass beads and submerged in liquid nitrogen. The sample is heated to $\sim 80^{\circ} \mathrm{C}$ and split into six different column/detector combinations housed in three gas chromatographs using UHP helium as the carrier gas: (1) DB-1 column (J\&W, Agilent, Santa Clara, CA, USA; $60 \mathrm{~m}, 0.32 \mathrm{~mm}$ I.D., $1 \mu \mathrm{m}$ film thickness) output to a flame ionization detector and a sulfur chemiluminescence detector; (2) DB-5 column (J\&W, $30 \mathrm{~m}, 0.25 \mathrm{~mm}$ I.D., $1 \mu \mathrm{m}$ film thickness) connected in series to a RESTEK 1701 column ( $5 \mathrm{~m}, 0.25 \mathrm{~mm}$ I.D., $0.5 \mu \mathrm{m}$ film thickness) and output to an electron capture detector; (3) RESTEK 1701 column (60 m, $0.25 \mathrm{~mm}$ I.D., $0.50 \mu \mathrm{m}$ film thickness) output to an electron capture detector; (4) PLOT column (J\&W GS-Alumina; $30 \mathrm{~m}, \quad 0.53 \mathrm{~mm}$ I.D.) connected in series to a DB-1 column (J\&W; $5 \mathrm{~m}, 0.53 \mathrm{~mm}$ I.D., $1.5 \mu \mathrm{m}$ film thickness) and output to an flame ionization detector; (5) DB-5ms column (J\&W; $60 \mathrm{~m}, 0.25 \mathrm{~mm}$ 
I.D., $0.5 \mu \mathrm{m}$ film thickness) output to a quadrupole mass selective detector (MSD, HP 5973). The MSD is set to operate in scan mode (SCAN) and selected ion monitoring mode simultaneously. Scan mode was used for identification, whereas selected ion monitoring (one ion chosen to quantify each compound) was used in order to achieve the maximum selectivity and to avoid potential interferences. All gas chromatographs and detectors used in this study were manufactured by Hewlett Packard (Palo Alto, CA, USA).

The first three of the seven longitudinal timepoints were analyzed globally without focused identification of a particular compound. After 2,3-butanedione was identified in the first two time-points, pure 2,3-butanedione was diluted to the parts per billion (ppb) level and then used as a qualitative standard, focusing the instrument for detection of 2,3-butanedione in the last four timepoints and for all time-points in the cross-sectional study. Quantification of 2,3-butanedione was carried out using both mass spectrometry and one of the flame ionization detectors.

\section{Statistical analyses}

Box-plots, linear regressions, analysis of variance and non-parametric tests were performed in $\mathrm{R}$ (v3.0.1) or JMP (v5.0.1.2).

\section{Results}

2,3-butanedione occurs in healthy and CF breath samples, and is affected by intravenous antibiotic therapy

Gas chromatography was used to monitor 2,3-butanedione concentrations longitudinally over seven time-points in one patient, and cross-sectionally at the same time-point in seven patients. These samplings always included simultaneous breath gas sampling of healthy volunteers in the same room, and background samples of room air. Volunteers were instructed to collect only the last two-thirds of each breath to avoid the gases produced by oral microbes.

A CF patient produced more 2,3-butanedione than the healthy control, except when receiving intravenous antibiotic therapy. In the longitudinal study one patient and a healthy control were simultaneously sampled approximately monthly. The non-CF and air controls contained between 3 and 2287 parts per trillion (ppt) 2,3-butanedione, whereas the CF samples contained between 1482 and $26751 \mathrm{ppt}$ (Figure 2 and Supplementary Table 3). In this longitudinal sample, 2,3-butanedione concentrations in $\mathrm{CF}$ versus non-CF were significantly different (repeated measures analysis of variance; $\mathrm{F}=336.7 ; P=0.0004)$. Most interestingly, the 2,3-butanedione concentration decreased over 10 -fold in this patient during treatment with

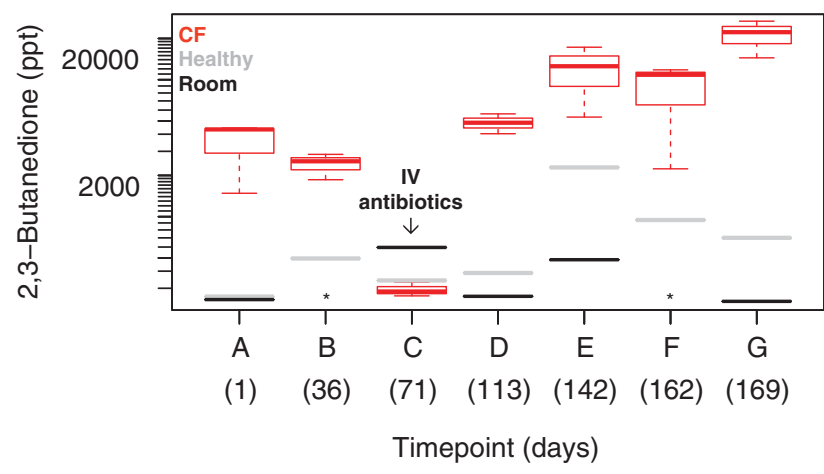

Figure 2 Box-plot showing concentrations of 2,3-butanedione from seven time-points taken over the course of 6 months in one CF patient (red), one gender matched healthy volunteer (gray) and room air samples (black). The red boxes represent the middle $50 \%$ of the data from the three CF breath samples taken at each timepoint, whereas the whiskers show the minimum and maximum values, and circles represent outliers. 2,3-butanedione levels on the $y$ axis are plotted on a logarithmic scale. The asterisks indicate room samples with a level of 2,3-butanedione that was undetectable (the 36-day time-point), or below the range of the $y$ axis (177ppt, 162-day time-point).

intravenous antibiotics (azithromycin, trimethoprim/ sulfamethoxazole and tobramycin) (Figure 2).

2,3-butanedione production varied significantly between $C F$ and non-CF volunteers. The results from our longitudinal study prompted us to examine 2,3-butanedione in additional $\mathrm{CF}$ and non-CF volunteers. CF patients harboring microbial communities dominated by different bacteria as indicated by clinical sputum culture data were chosen. This cross-sectional study sampled six CF patients in addition to the one patient followed longitudinally (Figure 2), four non-CF controls (each of which were sampled at the same time and in the same room as the matched CF patient), and ambient room air (Figure 3 and Supplementary Table 4). Only four non-CF volunteers donated breath samples because one volunteer donated samples (at different times) for multiple CF patients (CF2, CF4, CF5 and CF7 in Figure 3 and Table 1).

Four of the seven CF patients sampled (CF1-4) had elevated 2,3-butanedione concentrations in their breath samples compared with the simultaneous non-CF volunteers and room air samples. They were considered to be in a stable disease state at the time of sampling (Table 1). However, at the time of sampling patients CF6 and CF7 were undergoing antibiotic treatment for exacerbation, and patient CF5 was completing a 4 week course of intravenous tobramycin and aztreonam. These three patients were further classified as undergoing 'Acute' antibiotic therapy. Importantly, all of the patients regularly take antibiotics for maintenance, and those patients only undergoing maintenance therapy were classified as 'Suppressive' (Table 1).

Antibiotic therapy may affect 2,3-butanedione production in all patients. On the basis of the 
results from the longitudinal sampling, we examined the effect of antibiotic therapy on 2,3-butanedione production in this cross-sectional sample of CF and non-CF volunteers (Figure $3 \mathrm{~b}$ ). CF patients were classified as either being only on 'Suppressive' therapy, which is the maintenance dose of antibiotics all CF patients are prescribed during times of relatively good health, or 'Acute' therapy, which is

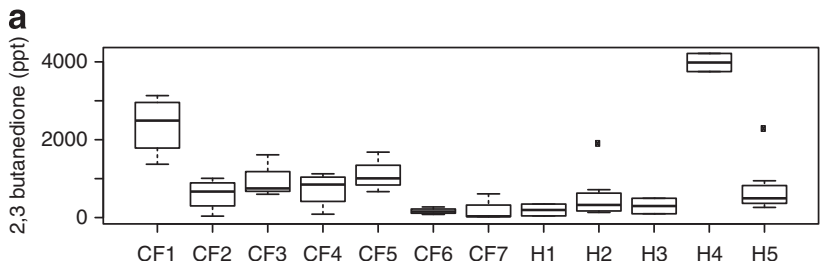
patient

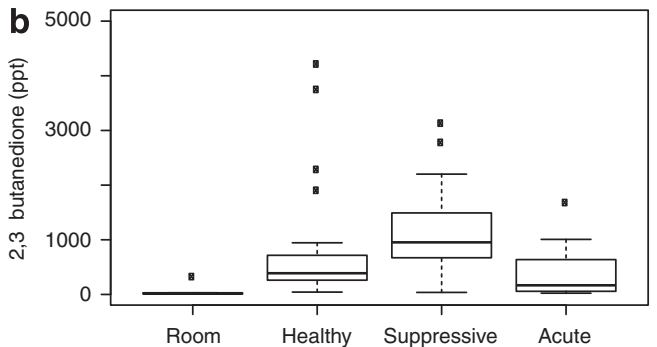

Figure 3 Box-plots of 2,3-butanedione concentrations in a crosssectional study of $\mathrm{CF}$ patients and paired non-CF volunteers. (a) Breath samples from seven CF patients and five non-CF volunteers showing 2,3-butanedione levels. Patient CF1 and H5 are the same individuals shown in Figure 2. Patient CF5 was completing a 4 week course of intravenous tobramycin and aztreonam at the time of sampling, and patients CF6 and CF7 were undergoing antibiotic treatment for exacerbation (see Table 1 for clinical information). (b) Box-plot of samples grouped by type of antibiotic therapy. typically intravenous administration of antibiotics during times of exacerbation or relatively poor health. Non-CF volunteers were not taking antibiotics, and were classified as 'Healthy'.

When all replicate measurements were analyzed as separate data points, patients undergoing 'Acute' versus 'Suppressive' antibiotic therapy had significantly different levels of 2,3-butanedione present (analysis of variance; $F=11.384 ; \quad P=0.00251$ ). 'Healthy' breath gases were intermediate (Figure 3b shows a box-plot of all measurements for each category). However, because replicate measurements were not combined for this analysis, we also performed the same analysis using the median value of replicate measurements. Using a single median value for each patient led to results that were statistically insignificant, though the trend was maintained. A power analysis suggested that the available sample size was insufficient to detect an effect: a sample size of 12 with an $\alpha=0.05$ had only 0.0961 power ( 1 is maximum). Obtaining significance at the $\alpha=0.5$ level would require 93 volunteers, and at the $\alpha=0.1$ level would require 72 volunteers.

\section{Metagenomes indicate potential for 2,3-butanedione production}

To identify microbial candidates that may be responsible for 2,3-butanedione production, we sequenced metagenomes from sputum induced in our cross-sectional CF volunteers, and one non-CF volunteer. Although our conclusions will be limited by this small sample size, complete metagenomes provide an enormous volume of data for generating new, and testing existing, hypotheses.

Table 1 Samples from CF and non-CF volunteers

\begin{tabular}{|c|c|c|c|c|c|c|c|c|}
\hline Study & Patient & Time-point & $\begin{array}{l}\text { Disease } \\
\text { severity }\end{array}$ & Clinical state & $\begin{array}{l}\text { Antibiotic } \\
\text { therapy }\end{array}$ & $\begin{array}{l}\text { Metagenome } \\
\text { data }\end{array}$ & $\begin{array}{l}\text { Breath gas } \\
\text { data }\end{array}$ & $\begin{array}{c}\text { Healthy } \\
\text { control }\end{array}$ \\
\hline Longitudinal & CF1 & A & Mild/moderate & Stable & Suppressive & No & Yes & H5 \\
\hline Longitudinal & CF1 & B & & Stable & Suppressive & No & Yes & H5 \\
\hline Longitudinal & CF1 & $\mathrm{C}$ & & Treatment & Acute & No & Yes & H5 \\
\hline Longitudinal & CF1 & $\mathrm{D}$ & & Stable & Suppressive & No & Yes & H5 \\
\hline Longitudinal & CF1 & $\mathrm{E}$ & & Stable & Suppressive & No & Yes & H5 \\
\hline Longitudinal & CF1 & $\mathrm{F}$ & & Stable & Suppressive & No & Yes & H5 \\
\hline Longitudinal & CF1 & $\mathrm{G}$ & & Stable & Suppressive & No & Yes & H5 \\
\hline Cross-sectional & CF1 & $\mathrm{H}$ & & Stable & Suppressive & Yes & Yes & H3 \\
\hline Cross-sectional & CF2 & $\mathrm{A}$ & Moderate & Stable & Suppressive & Yes & Yes & $\mathrm{H} 2$ \\
\hline Cross-sectional & CF3 & A & Moderate & Stable & Suppressive & Yes & Yes & H1 \\
\hline Cross-sectional & CF4 & A & Mild & Stable & Suppressive & Yes & Yes & $\mathrm{H} 2$ \\
\hline Cross-sectional & CF5 & A & Severe & Stable & Acute & Yes & Yes & $\mathrm{H} 2$ \\
\hline Cross-sectional & CF6 & A & Moderate & Treatment & Acute & Yes & Yes & $\mathrm{H} 4$ \\
\hline Cross-sectional & CF7 & A & Severe & Post treatment & Acute & Yes & Yes & $\mathrm{H} 2$ \\
\hline Cross-sectional & H1 & A & None & None & None & No & Yes & \\
\hline Cross-sectional & $\mathrm{H} 2$ & A & None & None & None & Yes & Yes & \\
\hline Cross-sectional & Н3 & A & None & None & None & No & Yes & \\
\hline Cross-sectional & $\mathrm{H} 4$ & A & None & None & None & No & Yes & \\
\hline Longitudinal & H5 & A & None & None & None & No & Yes & \\
\hline
\end{tabular}

Abbreviation: CF, cystic fibrosis. 
Genes for butanedione metabolism and phenazine production are present in sputa from CF patients. Microbial DNA was isolated and sequenced as described in the Materials and methods. The overall taxonomic distribution of each sample was determined through identification of sequences known to be unique to sequenced strains of bacteria using Metaphlan (Segata et al., 2012), revealing that polymicrobial infections in sputum samples in these patients are unique to each individual, and dominated by $R$. mucilaginosa, Streptococcus spp., and $P$. aeruginosa (Figure 4). Interestingly, the microbes found in the healthy volunteer were remarkably similar to those from the CF patients, being dominated by Rothia, Pseudomonas and Streptococcus. Although $R$. mucilaginosa was present in both CF and non-CF, the dominant Streptococcus spp. in CF samples were $S$. parasanguinis and $S$. salivarius, whereas the non-CF volunteer had S. mitis, $S$. oralis, $S$. infantis and $S$. australis.

The metagenomes were mined for genes related to acetoin biosynthesis, including acetolactate synthase (budB), acetolactate decarboxylase (budA) and butanediol dehydrogenase (budC; the metabolic pathways are summarized in Figure 1). A total of 6278 raw reads matched the known butanedione metabolism genes (BLASTn; e-value cutoff $1 \times 10^{-10}$, with at least $40 \mathrm{bp}$ query coverage and $40 \%$ sequence identity). The taxonomic distribution of these genes was distinct from that of the total community, by having an over-representation of Streptococcus spp. (Figure 5). The 466 reads with high quality hits to acetoin metabolism genes (BLASTn; $e$-value cutoff $1 \times 10^{-10}$, with at least $60 \mathrm{bp}$ query coverage and

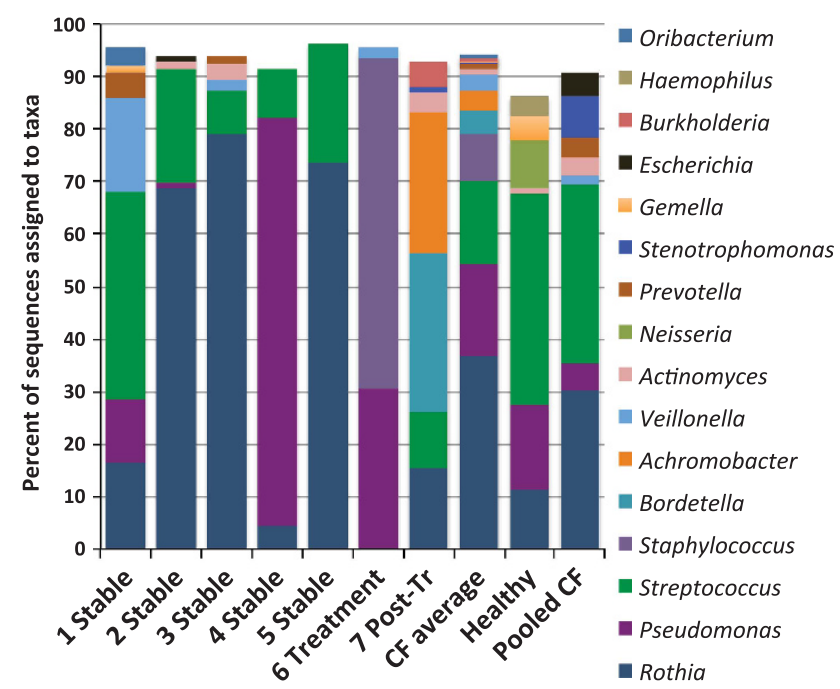

Figure 4 Taxonomy of microbial communities as assessed by Metaphlan for seven CF patients, from 13.5 million Ion Torrent reads of $\sim 140 \mathrm{bp}$ in length. The average fractional abundance of taxa for the seven CF patients from the cross-sectional study is also shown (CF average), in addition to the taxonomy of one sputum sample induced in a healthy volunteer (Healthy), and the pooled data from a previous data set taken from six CF patients at two to four time-points (Pooled CF) (Lim et al., 2012). Relative abundance data can be found in Supplementary Table 10.
$90 \%$ sequence identity) were heavily dominated by Streptococcus spp. (335 of 446 top hits), despite $P$. aeruginosa and $R$. mucilaginosa being numerically dominant in most patients. Reanalysis of 18 published metagenomes from six CF patients (Lim et al., 2012) confirmed the over-representation of Streptococcus-associated genes for butanedione metabolism (Supplementary Figure 1 and Supplementary Table 1). Fragment recruitment of metagenomic reads against the butanedione pathway genes from $S$. parasanguinus, a common species in these samples, is shown in Figure 6.

Streptococcus spp. were the only bacteria in these microbial communities that encoded all of the enzymes in the 2,3-butanedione pathway. budA was not present in $P$. aeruginosa (in these CF metagenomes from San Diego nor in publically available fully sequenced genomes (Winsor et al., 2011), and budC was not present in the highly abundant $R$. mucilaginosa. However, $R$. mucilaginosa and/or $P$. aeruginosa have the ability to consume 2,3-butanedione, as they encode $a c o A$ and $a c o B$, the genes whose products catabolize acetoin for use in central metabolism.

Electrical current production increases when $P$. aeruginosa is directly fed 2,3-butanediol or when it is cocultured with microbes that produce it (Venkataraman et al., 2011). Under these microaerobic conditions, $P$. aeruginosa increases its production of the redox active phenazine, pyocyanin (Venkataraman et al., 2011), which may act as an alternative electron acceptor (Cox, 1986; Wang and Newman, 2008; Wang et al., 2011). Therefore, to identify whether these microbial communities had the potential for this 2,3-butanediol-induced phenazine production, we mined the metagenome data for genes that encode phenazines. As shown in Figure 5, phenazine biosynthesis genes were present in the CF metagenomes (BLASTn; 4109 hits with $e$-value $1 \times 10^{-10}, 40 \mathrm{bp}$ length and $40 \%$ identity), the vast majority of which (95\%) were found in P. aeruginosa.

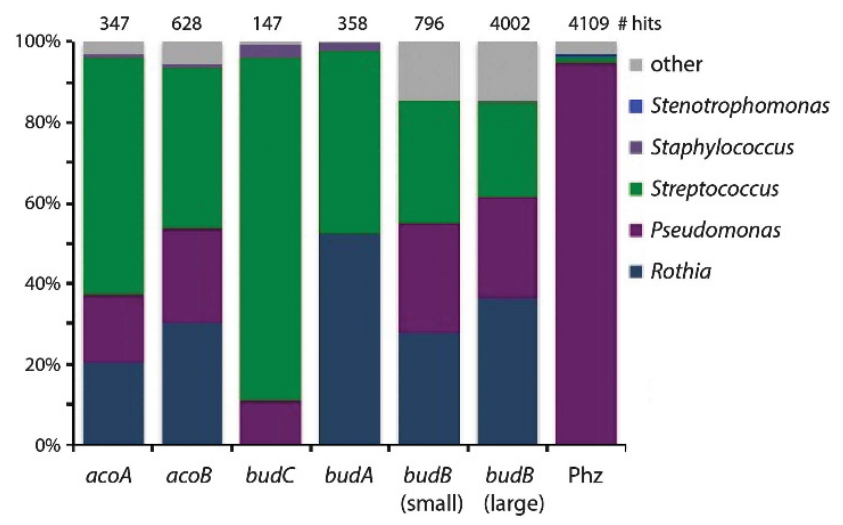

Figure 5 Taxonomy of BLASTn hits to genes involved in 2,3butanedione metabolism (6278 hits) and phenazine synthesis (4109 hits). budB small and budB large refer to short and long subunits of $b u d B$, respectively. 

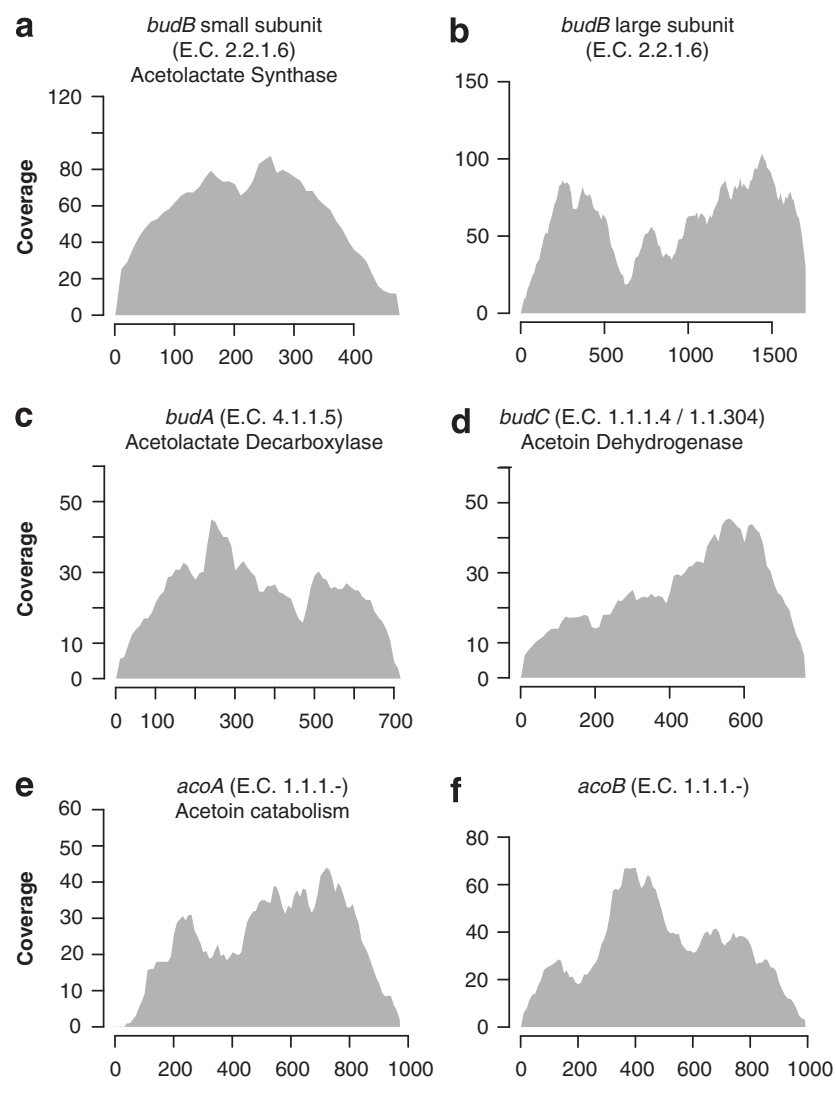

Position in gene (bp)

from Streptococcus parasanguinis ATCC 15912, complete genome

Figure 6 Fragment recruitment diagrams showing hits to Streptococcus parasanguinus acetoin metabolism genes from seven CF sputum microbial metagenomes. The number of hits at each position is shown for (a) the budB small subunit, also known as acetolactate synthase, (b) the budB large subunit, (c) budA or acetolactate decarboxylase, (d) budC or acetoin dehydrogenase, and the acetoin catabolism genes are shown in (e) $a c o A$ and (f) $a c o B$. Hits with a BLASTn $e$-value of $1 \times 10^{-10}, 40 \mathrm{bp}$ and $40 \%$ identity were retained.

Microbial abundance and 2,3-butanedione production. To identify how varying abundances of each candidate genus could predict the amount of 2,3-butanedione produced, we used linear models to identify how much variation in 2,3-butanedione concentration could be explained by the relative abundances of Streptococcus, Rothia and Pseudomonas in the CF patients. Only variability in Streptococcus could significantly predict differences in 2,3-butanedione concentration (Supplementary Figure 4: $\left.r^{2}=0.76 ; \quad P=0.01\right)$, whereas Rothia $\left(r^{2}=0.00 ; P=0.94\right)$ and Pseudomonas $\left(r^{2}=0.00\right.$; $P=0.97)$ abundances had no effect. Additional patient information, microbial abundances and a corroborating correlation analysis can be found in Supplementary Tables 8 and 9.

The effects of antibiotic treatment on microbial communities. The potential for 'Acute' antibiotic therapies to influence the production of 2,3-butanedione prompted us to examine how these therapies affect the microbial composition and physiology of lung communities. Particularly, we aimed to identify which genera were indicative of antibiotic therapy group membership. Using the LABDSV package in R, we attempted to identify genera that were significantly associated with 'Acute' or 'Suppressive' therapies. None of the genera in the metagenomic data set were significantly associated with patients undergoing 'Acute' or 'Suppressive' therapy. Either this analysis suffered from the same lack of power as the analysis of variance mentioned above, or the different antibiotic therapies do not predictably alter the underlying microbial communities. Thus, any effects antibiotic treatment may have on 2,3-butanedione production could be due to altering the physiology of the microbes present, rather than altering the abundances of individual microbes.

\section{Discussion}

The presence of 2,3-butanedione in breath indicates low $\mathrm{pH}$, low oxygen conditions and can serve as a cue to neighboring microbes. Here we have shown that 2,3-butanedione is abundant in CF patients, that its concentration varies with antibiotic therapy, and that it may be produced by Streptococcus species.

\section{2,3-butanedione in the breath gases}

Healthy people emit hundreds to thousands of breath gases in the ppb to parts per quadrillion concentration range (Pauling et al., 1971; Cao and Duan, 2006). These products are derived from: (i) exchange of human metabolic products from the blood across the lung epithelia; (ii) inhalation of gases from the environment, which may be altered enzymatically or absorbed in the lung; (iii) microbial metabolites produced in the airways or oral cavity (Kamboures et al., 2005; Scott-Thomas et al., 2010) and (iv) hydrocarbons that may derive from the breakdown of cell membranes by reactive oxygen species and other toxic molecules secreted in the context of inflammation (Robroeks et al., 2010). Breath gases can be used to identify the cause of an infection if the metabolite is unique to a particular species of bacteria, or the physiology of the microbial community if the metabolite is only produced under particular conditions, such as low $\mathrm{pH}$ or oxygen (Bos et al., 2013).

Healthy people can have a low background of 2,3-butanedione from intestinal and oral microbial metabolism (de Graaf et al., 2010). This may explain higher levels of 2,3-butanedione in healthy controls compared with the room signal. To mitigate the signal from these oral microbes as much as possible, we included non-CF volunteers, and all sampled individuals were instructed to discard the first few seconds of their breath sample.

In this study, volunteers were requested not to consume anything but water for $1 \mathrm{~h}$ before sampling, 
and reported what they had consumed in the hours leading up to sample collection. One non-CF volunteer who had high concentrations of breath 2,3-butanedione (H4) reported that he or she did not consume anything other than water in the $2 \mathrm{~h}$ before sampling, and that he or she did not have any respiratory infections or symptoms in the month preceding the sampling. The origin of 2,3-butanedione in this individual is unknown, and warrants further study of 2,3-butanedione concentrations in breath gases from a larger study population. In general, detection of 2,3-butanedione in the breath may derive from food or from the metabolism of microbes in the airways. It is not found in the blood, and when compared with the room air was found to be at a threefold higher concentration in the breath samples of 28 healthy volunteers who did not fast and remained still for $10 \mathrm{~min}$ in the same room where samples were collected (Mochalski et al., 2013). Alcohol metabolism may also lead to detectable levels of 2,3-butanediol in human blood and urine (Otsuka et al., 1999).

During a 6-month longitudinal study, the concentration of 2,3-butanedione was consistently elevated in CF1 with the exception of time-point C (Figure 2). The first two and the last four samples were taken during periods of stability for the CF patient. Timepoint $\mathrm{C}$ was the only one taken when the patient was in the hospital; breath sampling occurred 5 days after the patient began a course of acute antibiotic treatment. Furthermore, in the cross-sectional study, patients who had recently finished (CF5), or were undergoing (CF6 and CF7), acute treatment also had lower levels of 2,3-butanedione. 2,3-butanedione in breath samples taken after discarding the first third of the breath may indicate that oral microbes such as Streptococcus have moved into the lung. There, these immigrant microbes may influence the physiology of the microbial community, though their activities may be more sensitive to antibiotics than long-time residents of the lung such as $P$. aeruginosa. Decreases in 2,3-butanedione concentration during acute therapy could be attributed to a reduction in the density of the producing bacteria, a change in the metabolism of the bacterial community, or the consumption of this metabolite. Identifying whether a causative link exists between 2,3-butanedione production, antibiotic therapy and patient health is an important goal for future research with a larger cohort.

Streptococcus spp. likely produced 2,3-butanedione detected in the CF breath gases

The presence of 2,3-butanedione in the breath of these individuals suggests active production of 2,3butanedione. Our metagenome data combined with the presence of 2,3-butanedione in culture headspace samples (Filipiak et al., 2012a,b) indicate that Streptococcus spp. were most likely producing 2,3-butanedione in these samples. Activity of Streptococcus spp. in one patient, with significant 2,3-butanedione production, was suggested by metatranscriptome data from a previous study; however, sequence read coverage was too low to detect the presence of acetoin biosynthesis genes (Lim et al., 2012). Although the Pseudomonas in the samples described here did encode part of the acetoin biosynthesis pathway, they lacked the essential enzyme BudA (Figure 5). 2,3-butanedione can be produced spontaneously from acetolactate (Figure 1), however, Lactococcus mutants lacking acetolactate decarboxylase (BudA) do not produce nearly as much 2,3-butanedione (Aymes et al., 1999). This is consistent with previous reports showing $P$. aeruginosa does not produce 2,3-butanedione (Filipiak et al., 2012b).

The interplay between Streptococcus and Pseudomonas is important for a polymicrobial understanding of $\mathrm{CF}$ lung communities, because these bacteria usually colonize CF children and adults for the long term (Zemanick et al., 2011; Delhaes et al., 2012; Zhao et al., 2012). Streptococcous spp are considered to be generally dominant in the oral cavity of humans, comprising $>20 \%$ of the oral microbial communities that were sequenced as part of the human microbiome project (Bik et al., 2010; Grice and Segre, 2012). Likewise, $R$. mucilaginosa is a common although less dominant oral bacteria (Grice and Segre, 2012). Oral origin of lung infection is well accepted; aspiration of oral material is known to cause about half of Pneumonia in the general population (Bousbia et al., 2012); a similar mechanism may be even more important in CF.

In several recent studies using culture independent approaches, Streptococcus spp. are among the top 5-10 most abundant microbes in the CF sputum samples (Blainey et al., 2012; Filkins et al., 2012; Fodor et al., 2012; Goddard et al., 2012; Lim et al., 2012; Zhao et al., 2012; Supplementary Table 2). The Streptococcus milleri group (S. anginosus, $S$. constellatus, and $S$. intermedius) has been associated with exacerbations in a Canadian CF cohort and antibiotic treatment targeting this group resolved respiratory symptoms in the patients (Sibley et al., 2008). S. pneumoniae and its virulent phage Dp-1 were abundant in patient CF1 (Supplementary Figure 2) (Lim et al., 2012), and Streptococcus reads accounted for a relatively high percentage of all sequence reads from $\mathrm{CF}$ patients examined herein, further supporting the presence of Streptococcus spp. in CF microbial communities, though most clinical labs discard plates containing Streptococcus spp. and other typical members of the oral cavity as contaminants.

\section{2,3-butanedione and its potential effects on CF lung microbial community physiology}

Placing these results in the context of the phenazine literature, we propose a model of cross-feeding between 2,3-butanedione producers such as Streptococcus spp. and phenazine producing $P$. aeruginosa (Figure 7a). Cross-feeding could occur 


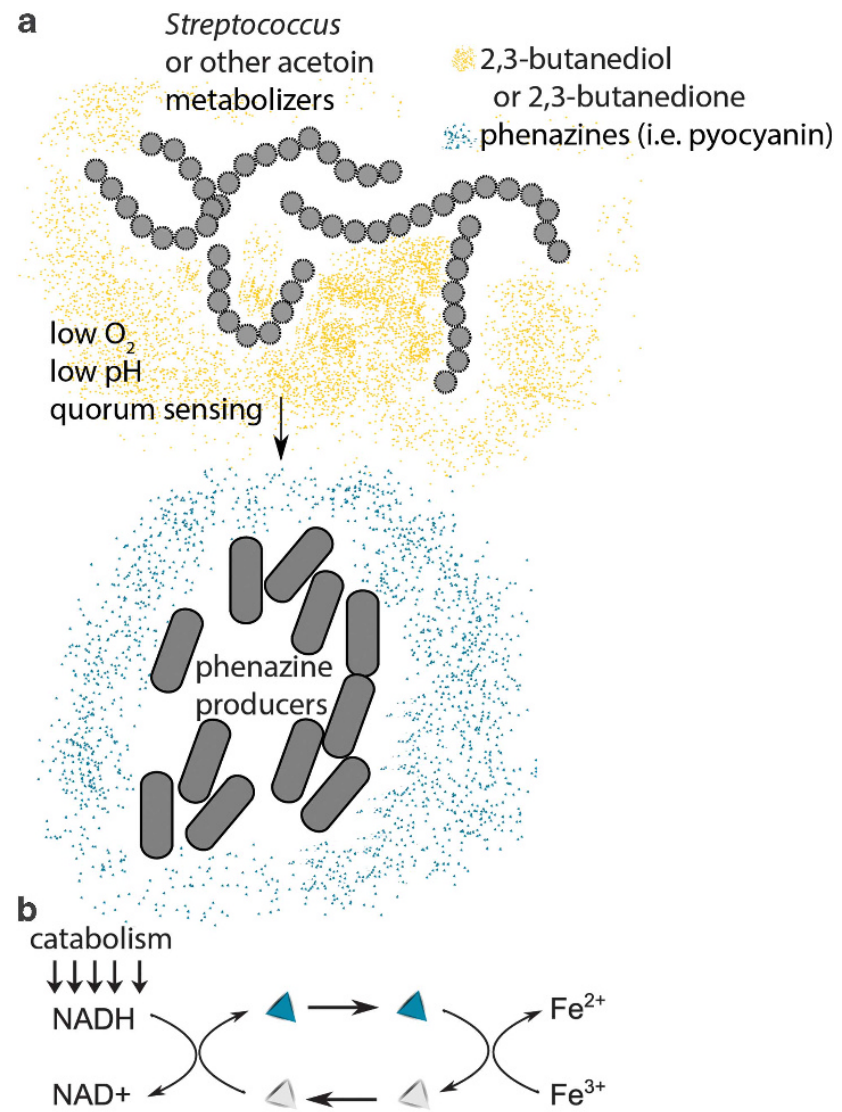

or other $\mathrm{e}^{-}$acceptor

Figure 7 Model for synergism between Streptococcus (or other butanediol producers) and Pseudomonas (or other phenazine producers). (a) In low $\mathrm{O}_{2}$, low $\mathrm{pH}$ and quorum sensing conditions, Streptococcus and some other bacteria activate their acetoin metabolism, producing 2,3-butanedione and 2,3-butanediol. This has been shown to elevate phenazine production in $P$. aeruginosa (Venkataraman et al., 2011). (b) In low $\mathrm{O}_{2}$, phenazines can act as an alternative electron acceptor, and $\mathrm{Fe}^{3+}$ or other electron acceptors could recycle the phenazine redox state in the absence of Oxygen. Blue and white triangles indicate reduced and oxidized phenazine molecules, respectively.

throughout the CF airways, as a volatile molecule such as 2,3-butanedione could easily travel through the airways, affecting microbes at greater distances. In response to 2,3-butanediol, $P$. aeruginosa produces phenazines (Venkataraman et al., 2011) that are known to increase in concentration as $\mathrm{CF}$ progresses (Hunter et al., 2012). The abundance of phenazines could then offer an alternative electron acceptor in oxygen limited conditions, thereby increasing 2,3-butanedione production and growth or survival of acetoin metabolizing strains (for example, Streptococcus spp.). Phenazine producing mutants of $P$. aeruginosa have been shown to form biofilms with architecture that increases surface area to increase access to oxygen, supporting the role of phenazines as alternative electron acceptors when access to oxygen is reduced (Dietrich et al., 2013). As shown in Figure 7b, phenazines that accept electrons from NADH that is produced in the course of microbial catabolism may recycle their redox state when they come into contact with $\mathrm{O}_{2}$ or $\mathrm{Fe}^{3+}$, depending on oxygen availability, $\mathrm{pH}$ and the redox potential of the phenazine (Wang and Newman, 2008).

Elucidating the relationship between the redox state of phenazines and $\mathrm{Fe}^{3+}$ is important because $\mathrm{CF}$ patients are often anemic, and high sputum iron content has been measured in CF patients during stable periods and exacerbations (Stites et al., 1998; Reid et al., 2007; Ghio et al., 2012; Gifford et al., 2012). The amount of iron sequestered in CF sputum is a considerable fraction of the total iron stores in the body, and is consistent with the possibility that iron in sputum originated from ferritin, which stores an average of $20-30 \%$ of the iron in a healthy adult (Lieu et al., 2001). Several studies of CF sputum have observed high iron and ferritin concentrations. The ferritin transcription in the San Diego CF patient's sputa (Supplementary Figure 3) is consistent with antioxidants controlling transcription of ferritin, and demonstrates the co-occurrence of ferritin transcription and high butanedione levels in the breath of the same CF patient. Under low oxygen conditions, nitrate or abundant $\mathrm{Fe}^{3+}$ could act as an alternative electron acceptor, recycling the redox state of the phenazines and maintaining a supply of $\mathrm{NAD}^{+}$(Figure 7b). Evidence supporting this mechanism can be found in recent studies of iron redox state in CF sputum, where an increasing fraction of iron was observed to be in the $\mathrm{Fe}^{2+}$ rather than the $\mathrm{Fe}^{3+}$ state as disease state progressed (Hunter et al., 2013). Because alternative electron acceptors provide a crucial survival advantage in the CF lung, limiting access to components that may contribute to their production-iron, butanedione or phenazines-could be a tool to control microbial infection and inflammation in the CF lung. For example, Gallium has been used successfully to interfere with $P$. aeruginosa iron metabolism (Kaneko et al., 2007).

\section{2,3-butanedione as a biomarker for Streptococcus activity and an anoxic lung environment}

A colorimetric assay to detect acetoin known as the Voges-Proskauer test was developed in Germany in the 1890s (Voges and Proskauer, 1898). Using this inexpensive assay, it may be possible to develop accessible methods for detecting the active, low $\mathrm{pH}$ metabolism of Streptococcus spp. and other butanedione-producing bacteria in respiratory patients. Detection of 2,3-butanedione in breath gases is important to better understand the resident bacterial community, and also has practical importance for lung health (Supplementary Tables 5 and 6). For example, microwave popcorn factory workers developed Bronchiolitis Obliterans after occupational exposure to 2,3-butanedione, leading to passage of the Popcorn Workers Lung Disease Prevention Act (H.R. 2693) in 2007 with recommendations to reduce exposure to inhaled 2,3-butanedione. The concentrations of 2,3-butanedione that 
were detected in our study are thought to be high enough to cause lung damage, and are above the proposed standard of $1 \mathrm{ppb}$ over an $8 \mathrm{~h}$ workday (Egilman et al., 2011).

Both 2,3-butanedione and 2,3-butanediol have been studied in a wide variety of host-microbe interactions (Supplementary Tables 5 and 6). 2,3butanedione has been shown to reduce the ability of mosquitos to detect $\mathrm{CO}_{2}$, preventing them from finding a host to prey on (Turner et al., 2011). In plant infections, microbial acetoin metabolism triggers the plant immune response, and is carefully timed to occur after cellulose degrading enzymes have already caused the damage needed for a successful infection (Effantin et al., 2011). One Vibrio cholera biotype secretes 2,3-butanediol and inhibits pro-inflammatory signals from epithelial cells (Bari et al., 2011), and 2,3-butanediol has also been shown to inhibit neutrophils in rats (Hsieh et al., 2007). The complexity of these interactions suggests that acetoin producing microbes and hosts have evolved together.

\section{Conclusions}

The physicians and spouses of some CF patients report that they can detect an odor associated with exacerbation (Cao and Duan, 2006). CF also leads to changes in the abundance of breath gases that are universally detected in human breath (Montuschi et al., 2012). In the case of infection, distinct gases that are unique to specific microbial metabolism or immune action may be present.

Linking together products unique to microbial metabolism from breath gas measurements with the genes detected by metagenomic sequences of microbial communities in sputum may enable development of biomarkers for early detection of exacerbations. Elucidation of the molecular signaling that occurs in CF microbial communities could also lead to new treatment strategies for altering microbial physiology. The detection of 2,3-butanedione in the breath of CF patients indicates: (i) active metabolism in the airways by 2,3-butanedione-producing bacteria, such as Streptococcus spp; (ii) synergism with other bacteria, where 2,3-butanedione acts as a carbon source and/or signaling molecule and (iii) 2,3-butanedione likely interacts with the host immune system, and causes direct physical damage through destruction of arginine side chains.

\section{Conflict of Interest}

The authors declare no conflict of interest.

\section{Acknowledgements}

We would like to thank Clarence C Lee and Timothy T Harkins from the Advance Applications Group, and Life Technologies for generously providing sequence data with
Ion Torrent technology. Anthony Fodor and Barbara Bailey provided statistics guidance. The clinical sampling coordination was greatly assisted by Doris Kwan. We thank Jeremy Barr for critical readings and discussions of the manuscript. This work was supported by the National Institute of Health through grants R01 GM095384-01 awarded to Forest Rohwer, and R01GM095384-01S1 to Katrine Whiteson.

\section{Data access}

Sequence data was depositied in the NCBI database under accession numbers SRX151603, SRX151605, SRX151606, 151607-151615, SRX106094, SRX 106095, SRX106105, SRX106106, SRX108115, SRX108128-SRX108130 for the 454 data, and Ion Torrent data can be found publically available in MG-RAST with ID\# 4541773.3.

\section{Author contributions}

KLW, SM, YWL, DRB, DC and FR designed research; KLW, SM and YWL performed research; KLW, SM, YWL, RQ and DC collected samples; KLW, SM, YWL and RS analyzed data and KLW, SM, HM and FR wrote the paper.

\section{References}

Aymes F, Monnet C, Corrieu G. (1999). Effect of alphaacetolactate decarboxylase inactivation on alphaacetolactate and diacetyl production by Lactococcus lactis subsp. lactis biovar diacetylactis. J Biosci Bioeng 87: 87-92.

Aziz RK, Devoid S, Disz T, Edwards RA, Henry CS, Olsen GJ et al. (2012). SEED servers: high-performance access to the SEED genomes, annotations, and metabolic models. PLoS One 7: e48053.

Bari W, Song Y-J, Yoon SS. (2011). suppressed induction of proinflammatory cytokines by a unique metabolite produced by Vibrio Cholerae O1 El Tor biotype in cultured host cells. Infect Immun 79: 3149-3158.

Bartowsky EJ, Henschke PA. (2004). The 'buttery' attribute of wine-diacetyl-desirability, spoilage and beyond. Int J Food Microbiol 96: 235-252.

Bik EM, Long CD, Armitage GC, Loomer P, Emerson J, Mongodin EF et al. (2010). Bacterial diversity in the oral cavity of 10 healthy individuals. ISME $J$ 4: 962-974.

Bilton D, Canny G, Conway S, Dumcius S, Hjelte L, Proesmans M et al. (2011). Pulmonary exacerbation: towards a definition for use in clinical trials. Report from the EuroCareCF Working Group on outcome parameters in clinical trials. J Cyst Fibros 10: S79-S81.

Blainey PC, Milla CE, Cornfield DN, Quake SR. (2012). Quantitative analysis of the human airway microbial ecology reveals a pervasive signature for cystic fibrosis. Sci Transl Med 4: 153ra130.

Bos LDJ, Sterk PJ, Schultz MJ. (2013). Volatile metabolites of pathogens: a systematic review. PLoS Pathog 9: e1003311. 
Bousbia S, Papazian L, Saux P, Forel JM, Auffray J-P, Martin C et al. (2012). Repertoire of intensive care unit pneumonia microbiota. PLoS One 7: e32486.

Cao W, Duan Y. (2006). Breath analysis: potential for clinical diagnosis and exposure assessment. Clin Chem 52: 800-811.

Colman JJ, Swanson AL, Meinardi S, Sive BC, Blake DR, Rowland FS. (2001). Description of the analysis of a wide range of volatile organic compounds in whole air samples collected during PEM-tropics A and B. Anal Chem 73: 3723-3731.

Conrad D, Haynes M, Salamon P, Rainey PB, Youle M, Rohwer F. (2012). Cystic fibrosis therapy: a community ecology perspective. Am J Respir Cell Mol Biol 48: 150-156.

Cox CD. (1986). Role of pyocyanin in the acquisition of iron from transferrin. Infect Immun 52: 263-270.

de Graaf AA, Maathuis A, de Waard P, Deutz NEP, Dijkema C, de Vos WM et al. (2010). Profiling human gut bacterial metabolism and its kinetics using [U-13C]glucose and NMR. NMR Biomed 23: 2-12.

Delhaes L, Monchy S, Fréalle E, Hubans C, Salleron J, Leroy $S$ et al. (2012). The airway microbiota in cystic fibrosis: a complex fungal and bacterial communityimplications for therapeutic management. PLoS One 7: e36313.

Dietrich LEP, Okegbe C, Price-Whelan A, Sakhtah H, Hunter RC, Newman DK. (2013). Bacterial community morphogenesis is intimately linked to the intracellular redox state. J Bacteriol 195: 1371-1380.

Dillies M-A, Rau A, Aubert J, Hennequet-Antier C, Jeanmougin M, Servant N et al. (2012). A comprehensive evaluation of normalization methods for Illumina high-throughput RNA sequencing data analysis. Brief Bioinform 14: 671-683.

Effantin G, Rivasseau C, Gromova M, Bligny R, Hugouvieux-Cotte-Pattat N. (2011). Massive production of butanediol during plant infection by phytopathogenic bacteria of the genera Dickeya and Pectobacterium. Mol Microbiol 82: 988-997.

Egilman DS, Schilling JH, Menendez L. (2011). A proposal for a safe exposure level for diacetyl. Int J Occup Environ Health 17: 122-134.

Filipiak W, Sponring A, Baur MM, Ager C, Filipiak A, Wiesenhofer $\mathrm{H}$ et al. (2012a). Characterization of volatile metabolites taken up by or released from Streptococcus pneumoniae and Haemophilus influenzae by using GC-MS. Microbiology 158: 3044-3053.

Filipiak W, Sponring A, Baur MM, Filipiak A, Ager C, Wiesenhofer $\mathrm{H}$ et al. (2012b). Molecular analysis of volatile metabolites released specifically by Staphylococcus aureus and Pseudomonas aeruginosa. BMC Microbiol 12: 113.

Filkins LM, Hampton TH, Gifford AH, Gross MJ, Hogan DA, Sogin ML et al. (2012). Prevalence of streptococci and increased polymicrobial diversity associated with cystic fibrosis patient stability. J Bacteriol 194: 4709-4717.

Fodor AA, Klem ER, Gilpin DF, Elborn JS, Boucher RC, Tunney MM et al. (2012). The adult cystic fibrosis airway microbiota is stable over time and infection type, and highly resilient to antibiotic treatment of exacerbations. PLoS One 7: e45001.

Ghio AJ, Roggli VL, Soukup JM, Richards JH, Randell SH, Muhlebach MS. (2012). Iron accumulates in the lavage and explanted lungs of cystic fibrosis patients. J Cyst Fibros 12: 390-398.
Gifford AH, Moulton LA, Dorman DB, Olbina G, Westerman M, Parker HW et al. (2012). Iron homeostasis during cystic fibrosis pulmonary exacerbation. Clin Transl Sci 5: 368-373.

Goddard AF, Staudinger BJ, Dowd SE, Joshi-Datar A, Wolcott RD, Aitken ML et al. (2012). Direct sampling of cystic fibrosis lungs indicates that DNA-based analyses of upper-airway specimens can misrepresent lung microbiota. Proc Natl Acad Sci USA 109: 13769-13774.

Goss CH, Burns JL. (2007). Exacerbations in cystic fibrosis. 1: Epidemiology and pathogenesis. Thorax 62: 360-367.

Grice EA, Segre JA. (2012). The human microbiome: our second genome*. Ann Rev Genomics Hum Genet 13: 151-170.

Hsieh S-C, Lu C-C, Horng Y-T, Soo P-C, Chang Y-L, Tsai Y-H et al. (2007). The bacterial metabolite 2,3-butanediol ameliorates endotoxin-induced acute lung injury in rats. Microbes Infect 9: 1402-1409.

Hunter RC, Klepac-Ceraj V, Lorenzi MM, Grotzinger H, Martin TR, Newman DK. (2012). Phenazine content in the cystic fibrosis respiratory tract negatively correlates with lung function and microbial complexity. Am J Respir Cell Mol Biol 47: 738-745.

Hunter RC, Asfour F, Dingemans J, Osuna BL, Samad T, Malfroot A et al. (2013). Ferrous iron is a significant component of bioavailable iron in cystic fibrosis airways. MBio 4: e00557-13.

Jay JM. (1982). Antimicrobial properties of diacetyl. Appl Environ Microbiol 44: 525-532.

Kamboures MA, Blake DR, Cooper DM, Newcomb RL, Barker M, Larson JK et al. (2005). Breath sulfides and pulmonary function in cystic fibrosis. Proc Natl Acad Sci USA 102: 15762-15767.

Kaneko Y, Thoendel M, Olakanmi O, Britigan BE, Singh PK. (2007). The transition metal gallium disrupts Pseudomonas aeruginosa iron metabolism and has antimicrobial and antibiofilm activity. J Clin Invest 117: 877-888.

Kim K-s, Lee S, Ryu C-M. (2013). Interspecific bacterial sensing through airborne signals modulates locomotion and drug resistance. Nat Commun 4: 1809.

Lieu PT, Heiskala M, Peterson PA, Yang Y. (2001). The roles of iron in health and disease. Mol Aspects Med 22: 1-87.

Lim YW, Schmieder R, Haynes M, Willner D, Furlan M, Youle $\mathrm{M}$ et al. (2012). Metagenomics and metatranscriptomics: windows on CF-associated viral and microbial communities. J Cystic Fibros 12: 154-164.

Lynch SV, Bruce KD. (2013). The cystic fibrosis airway microbiome. Cold Spring Harb Perspect Med 3: a009738.

Mathews JM, Watson SL, Snyder RW, Burgess JP, Morgan DL. (2010). Reaction of the butter flavorant diacetyl (2,3-butanedione) with $\mathrm{N}$ - $\alpha$-acetylarginine: a model for epitope formation with pulmonary proteins in the etiology of obliterative bronchiolitis. J Agric food Chem 58: 12761-12768.

McLean JS, Fansler SJ, Majors PD, McAteer K, Allen LZ, Shirtliff ME et al. (2012). Identifying low pH active and lactate-utilizing taxa within oral microbiome communities from healthy children using stable isotope probing techniques. PLoS One 7: e32219.

Mochalski P, King J, Klieber M, Unterkofler K, Hinterhuber H, Baumann M et al. (2013). Blood and breath levels of selected volatile organic compounds in healthy volunteers. Analyst 138: 2134-2145. 
Montuschi P, Paris D, Melck D, Lucidi V, Ciabattoni G, Raia V et al. (2012). NMR spectroscopy metabolomic profiling of exhaled breath condensate in patients with stable and unstable cystic fibrosis. Thorax 67: 222-228.

Neville MJ, Collins JM, Gloyn AL, McCarthy MI, Karpe F. (2011). Comprehensive human adipose tissue mRNA and microRNA endogenous control selection for quantitative real-time-PCR normalization. Obesity (Silver Spring) 19: 888-892.

Ojoo JC, Mulrennan SA, Kastelik JA, Morice AH, Redington AE. (2005). Exhaled breath condensate $\mathrm{pH}$ and exhaled nitric oxide in allergic asthma and in cystic fibrosis. Thorax 60: 22-26.

Otsuka M, Harada N, Itabashi T, Ohmori S. (1999). Blood and urinary levels of ethanol, acetaldehyde, and C4 compounds such as diacetyl, acetoin, and 2,3-butanediol in normal male students after ethanol ingestion. Alcohol 17: 119-124.

Pauling L, Robinson AB, Teranishi R, Cary P. (1971). Quantitative analysis of urine vapor and breath by gas-liquid partition chromatography. Proc Natl Acad Sci USA 68: 2374-2376.

Pezzulo AA, Tang XX, Hoegger MJ, Alaiwa MH, Ramachandran S, Moninger TO et al. (2012). Reduced airway surface $\mathrm{pH}$ impairs bacterial killing in the porcine cystic fibrosis lung. Nature 487: 109-113.

Reid DW, Carroll V, O’May C, Champion A, Kirov SM. (2007). Increased airway iron as a potential factor in the persistence of Pseudomonas aeruginosa infection in cystic fibrosis. Eur Respir J 30: 286-292.

Robroeks CMHHT, van Berkel JJBN, Dallinga JW, Jöbsis Q, Zimmermann LJI, Hendriks HJE et al. (2010). Metabolomics of volatile organic compounds in cystic fibrosis patients and controls. Pediatr Res 68: 75-80.

Sanders DB, Bittner RCL, Rosenfeld M, Hoffman LR, Redding GJ, Goss CH. (2010). Failure to recover to baseline pulmonary function after cystic fibrosis pulmonary exacerbation. Am J Respir Crit Care Med 182: $627-632$.

Sanders DB, Bittner RCL, Rosenfeld M, Redding GJ, Goss CH. (2011). Pulmonary exacerbations are associated with subsequent FEV1 decline in both adults and children with cystic fibrosis. Pediatr Pulmonol 46: 393-400.

Schmieder R, Edwards R. (2011a). Quality control and preprocessing of metagenomic datasets. Bioinformatics 27: 863-864.

Schmieder R, Edwards R. (2011b). Fast identification and removal of sequence contamination from genomic and metagenomic datasets. PLoS One 6: e17288.

Scott-Thomas AJ, Syhre M, Pattemore PK, Epton M, Laing R, Pearson J et al. (2010). 2-Aminoacetophenone as a potential breath biomarker for Pseudomonas aeruginosa in the cystic fibrosis lung. BMC Pulm Med 10: 56.

Segata N, Waldron L, Ballarini A, Narasimhan V, Jousson O, Huttenhower C. (2012). Metagenomic microbial community profiling using unique clade-specific marker genes. Nat Methods 9: 811-814.

Sibley CD, Parkins MD, Rabin HR, Duan K, Norgaard JC, Surette MG. (2008). A polymicrobial perspective of pulmonary infections exposes an enigmatic pathogen in cystic fibrosis patients. Proc Natl Acad Sci USA 105: 15070-15075.
Snyder EE, Kampanya N, Lu J, Nordberg EK, Karur HR, Shukla M et al. (2007). PATRIC: the VBI PathoSystems Resource Integration Center. Nucleic Acids Res 35: D401-D406.

Stites SW, Walters B, O'Brien-Ladner AR, Bailey K, Wesselius LJ. (1998). Increased iron and ferritin content of sputum from patients with cystic fibrosis or chronic bronchitis. Chest 114: 814-819.

Strausbaugh SD, Davis PB. (2007). Cystic fibrosis: a review of epidemiology and pathobiology. Clin Chest Med 28: 279-288.

Tate S, MacGregor G, Davis M, Innes JA, Greening AP. (2002). Airways in cystic fibrosis are acidified: detection by exhaled breath condensate. Thorax 57: 926-929.

Turner SL, Li N, Guda T, Githure J, Cardé RT, Ray A. (2011). Ultra-prolonged activation of CO2-sensing neurons disorients mosquitoes. Nature 474: 87-91.

Venkataraman A, Rosenbaum MA, Perkins SD, Werner JJ, Angenent LT. (2011). Metabolite-based mutualism between Pseudomonas aeruginosa PA14 and Enterobacter aerogenes enhances current generation in bioelectrochemical systems. Energy Environ Sci 4: 4550.

Voges DWO, Proskauer B. (1898). Beitrag zur Ernahrungs physiologie und zur differential diagnose der Bakterien der hamorrhagishen septicamie. Zeit fur Hyg 28: 20.

Wang Y, Newman DK. (2008). Redox reactions of phenazine antibiotics with ferric (hydr)oxides and molecular oxygen. Environ Sci Technol 42: 2380-2386.

Wang Y, Kern SE, Newman DK. (2010). Endogenous phenazine antibiotics promote anaerobic survival of Pseudomonas aeruginosa via extracellular electron transfer. J Bacteriol 192: 365-369.

Wang Y, Wilks JC, Danhorn T, Ramos I, Croal L, Newman DK. (2011). Phenazine-1-carboxylic acid promotes bacterial biofilm development via ferrous iron acquisition. J Bacteriol 193: 3606-3617.

Winsor GL, Lam DKW, Fleming L, Lo R, Whiteside MD, $\mathrm{Yu}$ NY et al. (2011). Pseudomonas genome database: improved comparative analysis and population genomics capability for Pseudomonas genomes. Nucleic Acids Res 39: D596-D600.

Worlitzsch D, Tarran R, Ulrich M, Schwab U, Cekici A, Meyer KC et al. (2002). Effects of reduced mucus oxygen concentration in airway Pseudomonas infections of cystic fibrosis patients. J Clin Invest 109: 317-325.

Yoon SS, Hennigan RF, Hilliard GM, Ochsner UA, Parvatiyar $\mathrm{K}$, Kamani MC et al. (2002). Pseudomonas aeruginosa anaerobic respiration in biofilms: relationships to cystic fibrosis pathogenesis. Dev Cell 3: 593-603.

Zemanick ET, Sagel SD, Harris JK. (2011). The airway microbiome in cystic fibrosis and implications for treatment. Curr Opin Pediatr 23: 319-324.

Zhao J, Schloss PD, Kalikin LM, Carmody LA, Foster BK, Petrosino JF et al. (2012). Decade-long bacterial community dynamics in cystic fibrosis airways. Proc Natl Acad Sci USA 109: 5809-5814.

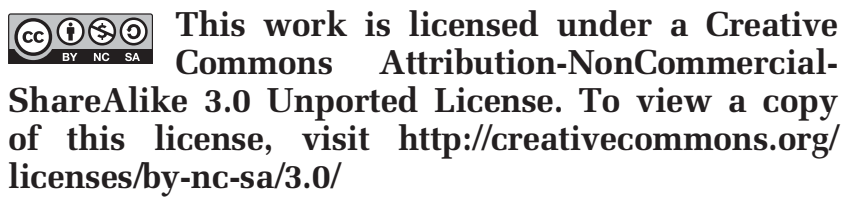

